Published in final edited form as:

J Am Chem Soc. 2016 October 26; 138(42): 13862-13865. doi:10.1021/jacs.6b09533.

\title{
Alcohols as Latent Coupling Fragments for Metallaphotoredox Catalysis: sp3-sp² Cross-Coupling of Oxalates with Aryl Halides
}

\author{
Xiaheng Zhang and David W. C. MacMillan* \\ Merck Center for Catalysis at Princeton University, Princeton, New Jersey 08544, United States
}

\begin{abstract}
Alkyl oxalates, prepared from their corresponding alcohols, are engaged for the first time as carbon radical fragments in metallaphotoredox catalysis. In this report, we demonstrate that alcohols, native organic functional groups, can be readily activated with simple oxalyl chloride to become radical precursors in a net redox-neutral $\mathrm{C}_{\mathrm{sp}^{3}}-\mathrm{C}_{\mathrm{sp}^{2}}$ cross-coupling with a broad range of aryl halides. This alcohol-activation coupling is successfully applied to the functionalization of a naturally occurring steroid and the expedient synthesis of a medicinally relevant drug lead.
\end{abstract}

\section{TOC Graphic}

Alcohols - native nucleophiles in cross-coupling via oxalyl chloride activation

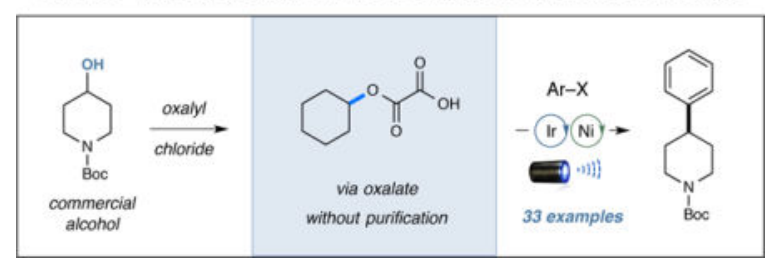

Over the last half-century, cross-coupling reactions, enabled mainly by $\mathrm{Pd}$ and $\mathrm{Ni}$ catalysts, have had a transformative impact on the synthetic chemistry community. ${ }^{1}$ The capacity to generically couple nucleophilic fragments (i.e., boronic acids, organozincs, organostannanes, Grignard reagents, etc.) with electrophilic partners (typically aryl or alkyl halides), with high regiospecificity and functional group tolerance has driven the wide-scale adoption of these methods within both industry and academia. Not surprisingly, practicality and cost issues have spurred ongoing efforts to develop new cross-coupling technologies that rely on naturally occurring, or native, organic functional groups as nucleophile partners in lieu of pregenerated, organometallic-based substrates.

One of the strategies used for engaging fragments bearing native functional groups in crosscouplings is the merger of nickel and photoredox catalysis, a form of metallaphotoredox. ${ }^{2}$ In this reactivity paradigm, the photoredox catalyst can generate a nucleophilic radical species

\footnotetext{
*Corresponding Author: dmacmill@princeton.edu.

Supporting Information

The Supporting Information is available free of charge on the ACS Publications website at DOI: (link to DOI)

Notes

The authors declare no competing financial interests.
} 
from the native functional group (nucleophile partner) as well as modulate the oxidation state of the nickel catalyst, which subsequently serves as a platform for reductive elimination and fragment coupling. Since 2014, our group and others have demonstrated that alkyl radical fragments generated from ubiquitous yet traditionally inert functionality (e.g., carboxylic acids, ${ }^{3}$ aliphatic $\mathrm{C}-\mathrm{H}$ bonds ${ }^{4}$ ) can be successfully coupled with aryl or alkyl halides to create unique and/or valuable $\mathrm{C}_{\mathrm{sp}^{3}}{ }^{-} \mathrm{C}_{\mathrm{sp}^{2}}$ and $\mathrm{C}_{\mathrm{sp}^{3}}-\mathrm{C}_{\mathrm{sp}^{3}}$ bonds.

Alcohols are amongst the most widely occurring, naturally abundant organic compounds known ${ }^{5}$ and in many cases are considered feedstock chemicals. On this basis we recently initiated a program to determine if $\mathrm{C}_{\mathrm{sp}^{3}}-\mathrm{OH}$ bonds could be activated to form nucleophilic partners for metallaphotoredox-based coupling technologies. The advantages of such a method are readily appreciated in that a range of alcohols, from simple to complex, are commercially available, whereas the corresponding boronic acids or esters are not yet broadly accessible or cost effective. Recently, the Overman group, in collaboration with our lab, reported that relatively strong $\mathrm{C}-\mathrm{O}$ bonds $(\sim 96 \mathrm{kcal} / \mathrm{mol})^{6} \mathrm{can}$ be generically cleaved to access $\mathrm{C}_{\mathrm{sp}}{ }_{3}$ radical fragments from alkyl oxalates under photoredox conditions. ${ }^{7,8}$ With this in mind, we questioned whether alkyl radicals generated in a similar manner could be merged with our metallaphotoredox platform as a means to expediently produce $\mathrm{C}-\mathrm{C}$ bonds from $\mathrm{C}-\mathrm{O}$ functionality. Moreover, we hoped to employ alcohols as the formal coupling partner via the rapid formation of alkyl oxalates (with inexpensive oxalyl chloride and without purification) prior to addition of the nickel catalyst or electrophile partner. Notably,

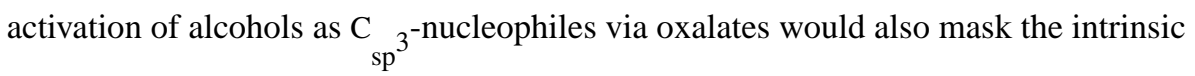
reactivity of the alcohols towards $\mathrm{C}-\mathrm{O}$ cross-coupling to form ethers. ${ }^{9}$ Herein we report our efforts to develop the first general route for efficiently converting a variety of alcohols via their corresponding oxalates into $\mathrm{C}_{\mathrm{sp}^{3}}-\mathrm{C}_{\mathrm{sp}^{2}}$ coupled products with aryl halides by way of the synergistic action of nickel and photoredox catalysis (Figure 1). ${ }^{10,11}$

The mechanistic details of our proposed transformation are outlined in Scheme 1. Photoexcitation with visible light of the iridium(III) photocatalyst, $\operatorname{Ir}[\mathrm{dFppy}]_{2}$ (dtbbpy) $\mathrm{PF}_{6}$ (1), is known to produce an excited state, ${ }^{*} \mathrm{Ir}^{\mathrm{III}} \mathbf{2}$, which is a strong oxidant $\left(E_{1 / 2}{ }^{\mathrm{red}}\left[{ }^{*} \mathrm{Ir}^{\mathrm{III}} / \mathrm{Ir}^{\mathrm{II}}\right]\right.$ $=+1.1 \mathrm{~V}$ vs. saturated calomel electrode $(\mathrm{SCE})$ in $\mathrm{MeCN}) .{ }^{12}$ Based on the previous work by Overman and collaborators, the oxidation of the oxalate after deprotonation of $\mathbf{3}\left(E_{\mathrm{p} / 2}=\right.$ $+1.26 \mathrm{~V}$ vs. $\mathrm{SCE}$ in $\mathrm{MeCN}$ for the cesium oxalate derivative of $\mathrm{N}$-Boc-4hydroxypiperidine) ${ }^{13}$ via single-electron transfer (SET) should be thermodynamically feasible, generating the reduced $\operatorname{Ir}^{\mathrm{II}}$ species $\mathbf{5}$ and the alkyl radical $\mathbf{4}$ - via successive loss of two equivalents of $\mathrm{CO}_{2}$. At the same time, oxidative addition of aryl bromide 7 to $\mathrm{L}_{\mathrm{n}} \mathrm{Ni}^{0}$ species 6 will form the aryl- $\mathrm{Ni}^{\mathrm{II}}$ species 8 , which should be rapidly intercepted by radical 4 to form the resulting $\mathrm{Ni}^{\mathrm{III}}$ complex 9 . At this stage, reductive elimination should then enable the desired $\mathrm{C}_{\mathrm{sp}^{3}}-\mathrm{C}_{\mathrm{sp}^{2}}$ bond formation to deliver the coupled product $\mathbf{1 0}$ and $\mathrm{Ni}^{\mathrm{I}}$ species $\mathbf{1 1}$. Finally, the two catalytic cycles merge via electron transfer from the reduced $1 \mathrm{r}^{\mathrm{II}}$ species 5 $\left(E_{1 / 2}{ }^{\mathrm{red}}\left[1 \mathrm{r}^{\mathrm{III}} / 1 \mathrm{r}^{\mathrm{II}}\right]=-1.46 \mathrm{~V} \text { vs. saturated calomel electrode }(\mathrm{SCE}) \text { in } \mathrm{MeCN}\right)^{12}$ to the $\mathrm{Ni}^{\mathrm{I}}$ 
complex $\left(\mathbf{1 1}, E_{1 / 2}{ }^{\text {red }}\left[\mathrm{Ni}^{\mathrm{II}} / \mathrm{Ni}^{0}\right]=-1.2 \mathrm{~V} \text { vs. SCE in DMF }\right)^{14}$ thereby regenerating both photocatalyst 1 and the $\mathrm{Ni}^{0}$ catalyst 6 .

Our investigation into this new coupling reaction began with exposure of the oxalate of $\mathrm{N}$ Boc-4-hydroxypiperidine (formed using oxalyl chloride, water and without purification) to methyl 4-bromobenzoate, photocatalyst $\mathbf{1}, \mathrm{NiBr}_{2} \mathrm{dtbbpy}(\mathbf{1 2})$, and a blue LED lamp in the presence of $\mathrm{CsHCO}_{3}$ as base. 1nitial experiments revealed a significant solvent effect, as the desired coupling adduct was obtained only in tetrahydropyran (THP) (Table 1, entry 2, 8\% yield), along with an ester side-product that arises from mono-decarboxylation of the oxalate substrate. ${ }^{15}$ Notably, an improvement in efficiency was obtained using DMSO as a cosolvent (entry 3,39\% yield). Next, we hypothesized that increased temperature should improve the rate of the desired double decarboxylation step. 1ndeed, superior yields, along with minimal quantities of ester formation, were obtained when the transformation was performed at $70{ }^{\circ} \mathrm{C}$ (entry $5,65 \%$ yield). 1 t should be noted that comparable yields are also possible when 1,4-dioxane is employed as the reaction medium in lieu of THP. Control experiments conducted in the absence of photocatalyst, nickel catalyst, base, or light resulted in no product, emphasizing the crucial role of all these components in the dual catalytic cycle.

With these optimized conditions in hand, we next began an exploration of the scope of the aryl bromide component. As shown in Table 2, aryl rings that incorporate a large variety of functional groups are readily tolerated. 1n summary, electron-deficient aryl bromides bearing carbonyl, sulfonyl, trifluoromethyl, and nitrile moieties provide good yields (> 55\%, products 13-21), while the coupling of electron-rich 4-bromotoluene was achieved with moderate efficiency $(\mathbf{2 2}, 42 \%$ yield). Given the importance of nitrogen-containing heterocycles in the production of bioactive molecules, we were delighted to find that a diverse array of substituted pyridyl bromides readily participate in this new coupling (products 23-28). Chemoselective oxidative addition to the bromide substituent was observed when 2-chloro-4-bromopyridine was employed to generate $\mathbf{2 4}$ in 56\% yield.

We next sought to explore the generality of this transformation with respect to the alcohol and the corresponding oxalate adduct. Further underscoring the practicality of this method, all oxalates were obtained by treatment of the alcohol with oxalyl chloride, which upon aqueous work-up was employed directly (and without purification) in the metallaphotoredox protocol. Oxalates derived from secondary alcohols were found to be generically successful in this transformation. For example, $N$-Boc-piperidinyl, cyclohexyl, and tetrahydropyranyl oxalates as well as larger ring oxalates were found to readily undergo fragment coupling (45-62\% yield, products 29-32). Moreover, 5-membered ring oxalates, including $\mathrm{N}$-Bocpyrrolidine and tetrahydrofuran derivatives were also effective nucleophile partners (52-78\% yield, products 33-36). Notably, oxalates derived from acyclic secondary alcohols provide the desired adducts (e.g., 37, 63\% yield). The union of primary alcohol-derived oxalates with aryl rings can also be accomplished using this metallaphotoredox dual catalysis method (37-95\% yield, products 38-42). Despite moderate efficiency in some cases (with the exception of benzyl alcohols), we anticipate that these alcohol substrates will find broad utility throughout medicinal chemistry studies. As expected, when the oxalate of cyclopropanemethanol was employed, ring opening was observed, consistent with a radical 
intermediate, ${ }^{16}$ to generate the homoallyl-bearing product $\mathbf{4 2}$. Complex oxalates were also successfully employed, showcasing the wide utility of this transformation. For example, substituted $N$-Boc-proline methyl ester and highly substituted cyclohexyl fragments were coupled with methyl 4-bromobenzoate to provide adducts $\mathbf{4 3}$ and $\mathbf{4 4}$ in useful yields and in good d.r. (7:1 and > 20:1, respectively). Additionally, adduct $\mathbf{4 5}$ was obtained from the oxalate of a naturally occurring steroid, further emphasizing the potential of employing native functionality to access new structural analogues or to perform late stage functionalization.

Last, to emphasize the synthetic value of using alcohols as $\mathrm{C}_{\mathrm{sp}}{ }^{3}$-nucleophiles, we applied this metallaphotoredox protocol to the synthesis of $\mathbf{4 6}$, a valuable intermediate used in the production of Q203, a promising candidate for the treatment of tuberculosis. ${ }^{17}$ As summarized in Figure 2, the 4-aryl- $N$-Boc-piperidine adduct was obtained in good yield using the optimized oxalate coupling conditions. After removal of the Boc group, the secondary amine was subsequently coupled with 4-cyanophenyl bromide using our previously published metallaphoto amination protocol ${ }^{18}$ to deliver precursor 46 in excellent yield.

\section{Supplementary Material}

Refer to Web version on PubMed Central for supplementary material.

\section{Acknowledgments}

Support was provided by NIH NIGMS R01 01 GM093213-01. X. Z. is grateful for a postdoctoral fellowship from the Shanghai Institute of Organic Chemistry. Patricia Zhang, Tracy Liu, and Andrei Iosub are thanked for their help in preparing this manuscript.

\section{References}

1. (a) Beller, M, Bolm, C. Transition Metals for Organic Synthesis. Vol. 1. Wiley-VCH; Weinheim: 2004. (b) de Meijere, A, Diederich, F. Metal-Catalyzed Cross-Coupling Reactions. 2nd. WileyVCH; Weinheim: 2004.

2. (a)Chu L, Lipshultz JM, MacMillan DWC. Angew Chem, Int Ed. 2015; 54:7929.(b)Kalyani D, McMurtrey KB, Neufeldt SR, Sanford MS. J Am Chem Soc. 2011; 133:18566. [PubMed: 22047138] (c)Sahoo B, Hopkinson MN, Glorius F. J Am Chem Soc. 2013; 135:5505. [PubMed: 23565980] (d)Shu XZ, Zhang M, He Y, Frei H, Toste FD. J Am Chem Soc. 2014; 136:5844. [PubMed: 24730447] (e)Tellis JC, Primer DN, Molander GA. Science. 2014; 345:433. [PubMed: 24903560]

3. (a)Zuo Z, Ahneman DT, Chu L, Terrett JA, Doyle AG, MacMillan DWC. Science. 2014; 345:437. [PubMed: 24903563] (b)Noble A, McCarver SJ, MacMillan DWC. J Am Chem Soc. 2015; 137:624. [PubMed: 25521443] (c)Zuo Z, Cong H, Li W, Choi J, Fu GC, MacMillan DWC. J Am Chem Soc. 2016; 138:1832. [PubMed: 26849354] (d)Johnston CP, Smith RT, Allmendinger S, MacMillan DWC. Nature. 2016; 536:322. [PubMed: 27535536]

4. (a)Jeffrey JL, Terrett JA, MacMillan DWC. Science. 2015; 349:1523.(b)Shaw MH, Shurtleff VW, Terrett JA, Cuthbertson JD, MacMillan DWC. Science. 2016; 352:1304. [PubMed: 27127237] (c)Joe CL, Doyle AG. Angew Chem, Int Ed. 2016; 55:4040.

5. Henkel T, Brunne RM, Muller H, Reichel F. Angew Chem, Int Ed. 1999; 38:643.

6. Pedley, JB, Naylor, RD, Kirby, SP. Thermochemical Data of Organic Compounds. 2nd. Chapman and Hall; New York: 1986. 
7. Nawrat CC, Jamison CR, Slutskyy Y, MacMillan DWC, Overman LE. J Am Chem Soc. 2015; 137:11270. [PubMed: 26322524]

8. From N-phthalimidoyl oxalates, see:Lackner GL, Quasdorf KW, Overman LE. J Am Chem Soc. 2013; 135:15342. [PubMed: 24074152]

9. (a) Hartwig, JF. Organotransition Metal Chemistry. 1st. University Science Books; USA: 2009. (b)Terrett JA, Cuthbertson JD, Shurtleff VW, MacMillan DWC. Nature. 2015; 524:330. [PubMed: 26266976]

10. for Ni-catalyzed reductive couplings of alkyl sulfonates, carbamates, or pivalates, see:(a)Harris MR, Hanna LE, Greene MA, Moore CE, Jarvo ER. J Am Chem Soc. 2013; 135:3303. [PubMed: 23414579] (b)Konev MO, Hanna LE, Jarvo ER. Angew Chem, Int Ed. 2016; 55:6730.(c)Liang Z, Xue W, Lin K, Gong H. Org Lett. 2014; 16:5620. [PubMed: 25333482] (d)Molander GA, Traister KM, O’Neill BT. J Org Chem. 2015; 80:2907. [PubMed: 25711834] for Cu-catalyzed reductive couplings, see:(e)Liu JH, Yang CT, Lu XY, Zhang ZQ, Xu L, Cui M, Lu X, Xiao B, Fu Y, Liu L. Chem Eur J. 2014; 20:15334. [PubMed: 25308802]

11. for Ni-catalyzed cross-coupling reactions of benzylic mesylates, methyl ethers and carbamates with organometallic reagents, see:(a)Do HQ, Chandrashekar ERR, Fu GC. J Am Chem Soc. 2013; 135:16288. [PubMed: 24164502] (b)Tobisu M, Yasutome A, Kinuta H, Nakamura K, Chatani N. Org Lett. 2014; 16:5572. [PubMed: 25325885] (c)Harris MR, Hanna LE, Greene MA, Moore CE, Jarvo ER. J Am Chem Soc. 2013; 135:3303. [PubMed: 23414579]

12. Singh A, Teerdigan K, Kelly M, Prasad KS, Krishnan S, Weaver JD. J Organomet Chem. 2015; 776:51.

13. See Supporting Information for measurement details.

14. Durandetti M, Devaud M, Périchon J. New J Chem. 1996; 20:659.

15. See Supporting Information for characterization data.

16. Biswas S, Weix DJ. J Am Chem Soc. 2013; 135:16192. [PubMed: 23952217]

17. Tang J, Wang B, Wu T, Wan J, Tu Z, Njire M, Wan B, Franzblauc SG, Zhang T, Lu X, Ding K. ACS Med Chem Lett. 2015; 6:814. [PubMed: 26191372]

18. Corcoran EB, Pirnot MT, Lin S, Dreher SD, DiRocco DA, Davies IW, Buchwald SL, MacMillan DWC. Science. 2016; 353:279. [PubMed: 27338703] 

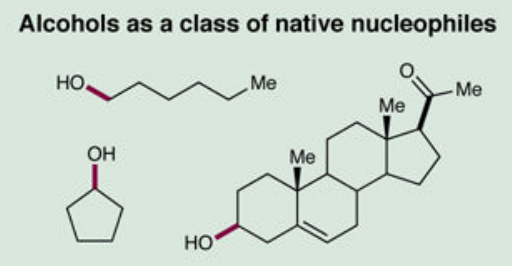

E simple abundant $\quad$ commercial
Challenge: $\mathrm{C}-\mathrm{O}$

bond activation<smiles>CCCC1(O)CCCCC1</smiles>

$96 \mathrm{kcal} / \mathrm{mol}$

high energetic barrier to cleavage

Metallaphotoredox-mediated alcohol cross-coupling via oxalates

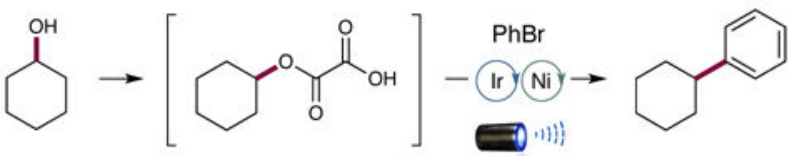

alcohol add oxalyl chloride, cross-coupled without purification product

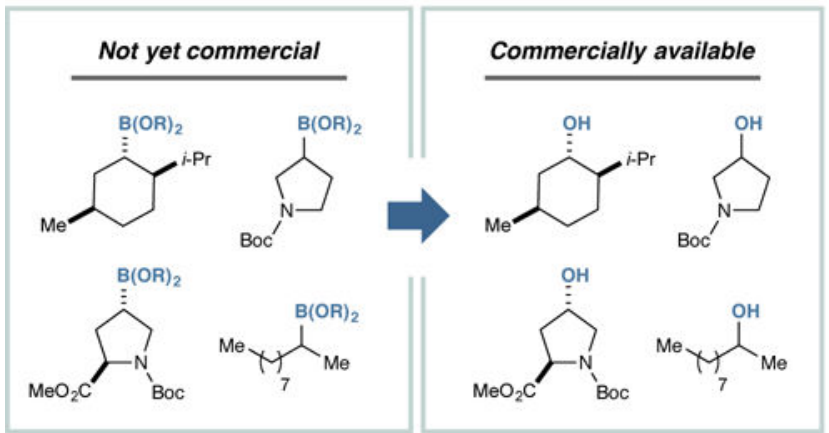

Figure 1.

$\mathrm{C}-\mathrm{C}$ Coupling of Alcohols via Oxalate Activation. 


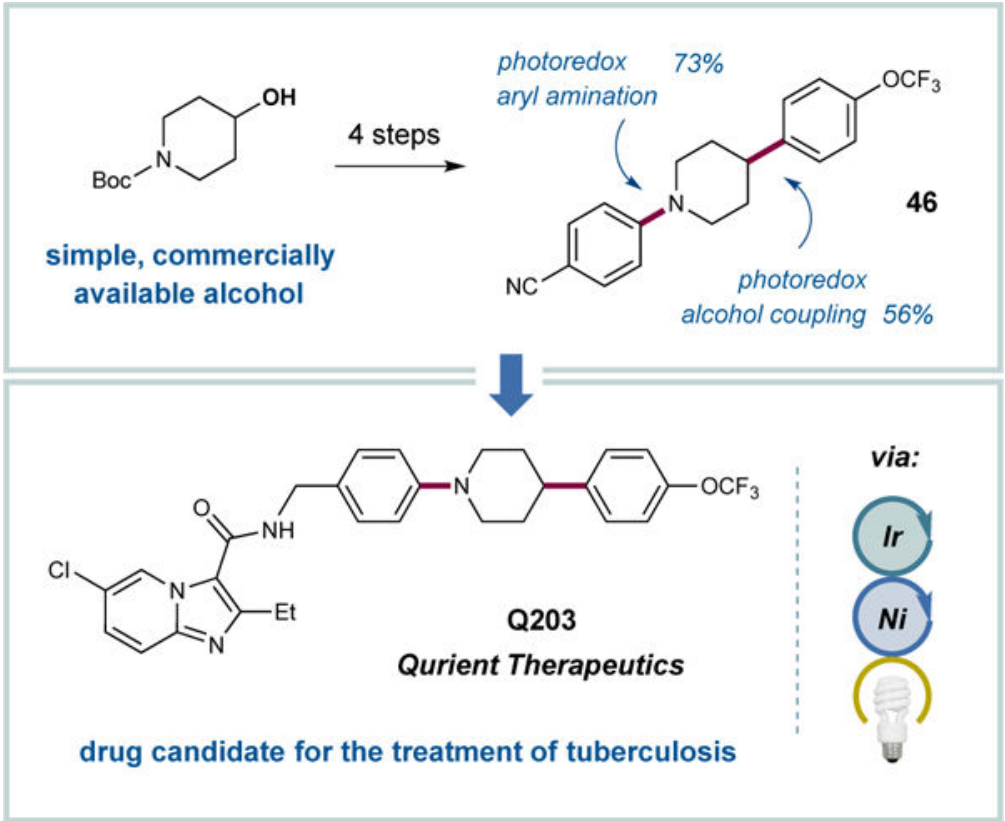

Figure 2.

Synthesis of a Precursor to Q203 via Sequential Visible Light Photoredox C-C and C-N Coupling Reactions. 


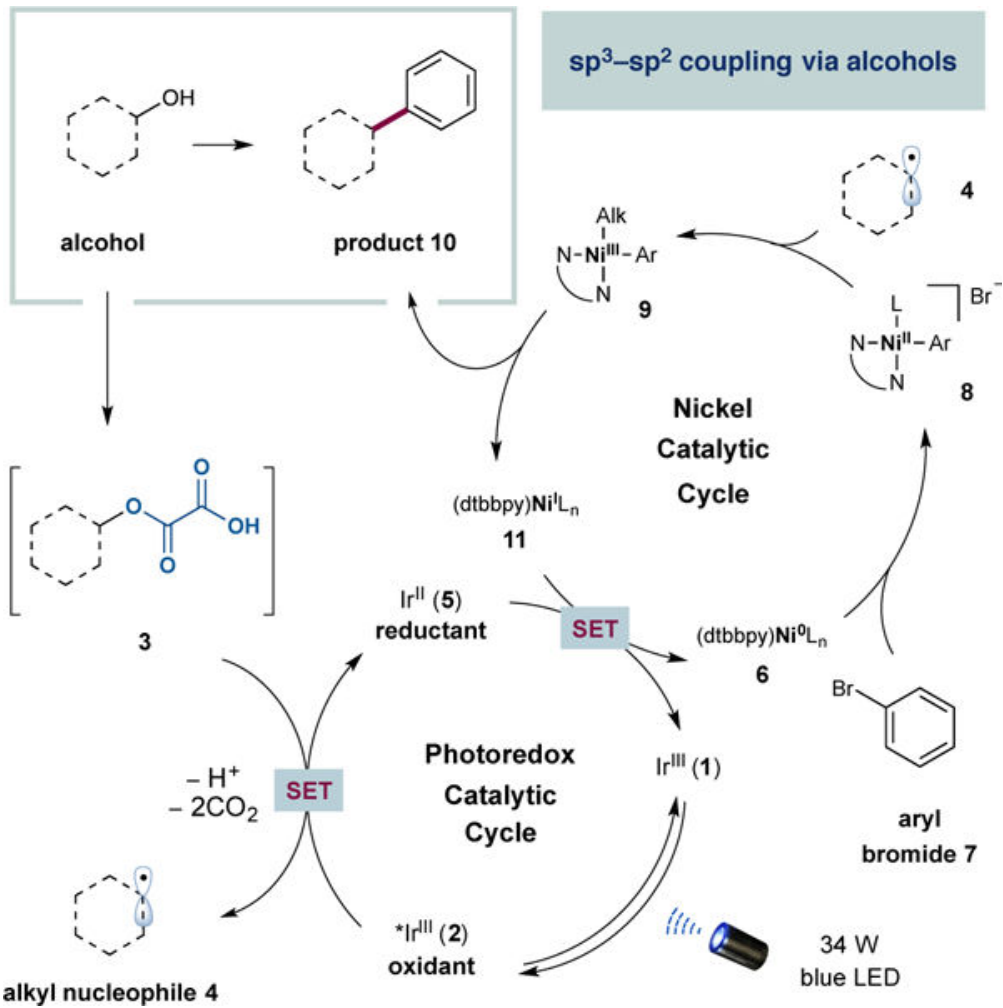

Scheme 1.

Steps of Proposed Metallaphotoredox Reaction. 


\section{Table 1}

Optimization of the Oxalate Cross-Coupling. ${ }^{a}$

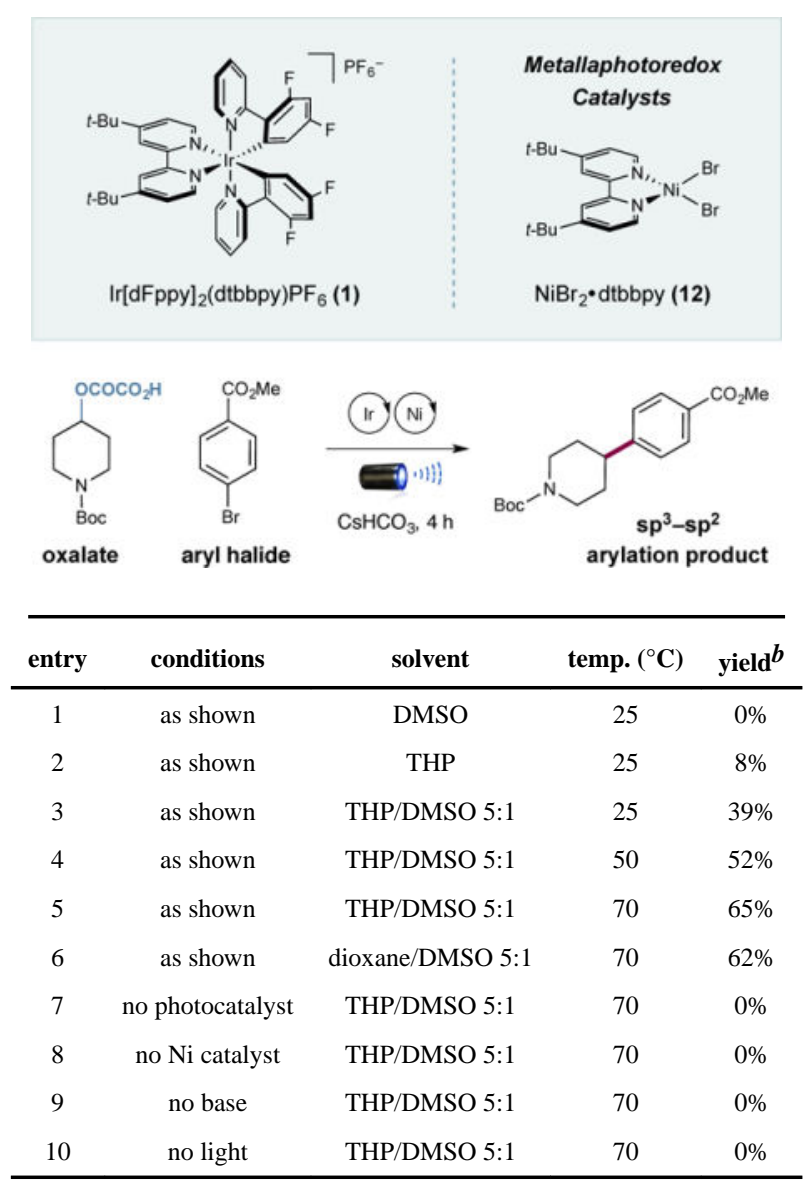

${ }^{a}$ Performed with photocatalyst 1 ( 1 mol $\left.\%\right)$, NiBr2 dtbbpy 12 (5 mol\%), aryl halide (1.0 equiv), oxalate (1.3 equiv) and $\mathrm{CsHCO}_{3}$ (1.5 equiv).

${ }^{b}$ Yields were obtained by ${ }^{1} \mathrm{H}$ NMR analysis of the crude reaction mixtures using an internal standard. 


\section{Table 2}

From Alcohols to Alkyl-Aryl Products: Scope of Metallaphotoredox Reaction of Oxalates with Aryl Bromides. $^{a}$

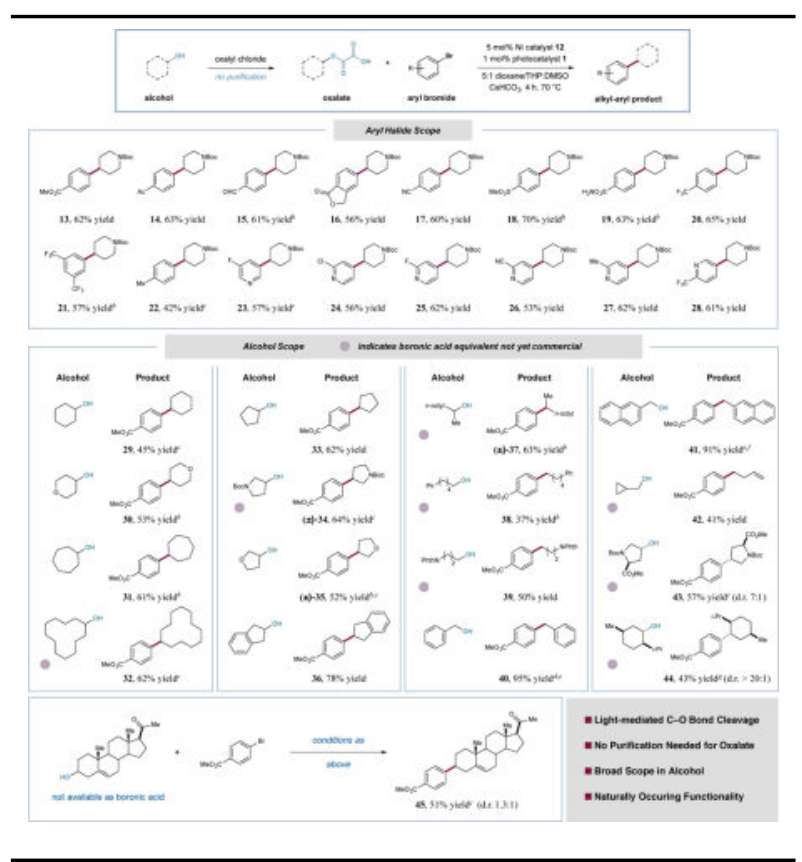

${ }^{a}$ All yields are isolated. Performed with photocatalyst 1 ( 1 mol\%), NiBr2 dtbbpy 12 (5 mol\%), aryl halide (1.0 equiv), oxalate (1.3 equiv), and $\mathrm{CsHCO}_{3}$ (1.5 equiv). Ratios of diastereomers determined by ${ }^{1} \mathrm{H}$ NMR. For detailed experimental procedures, see Supporting Information.

$b_{80}{ }^{\circ} \mathrm{C}$.

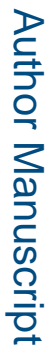

$c_{10 \mathrm{~mol} \%} \mathbf{1 2}$.

$d_{25}{ }^{\circ} \mathrm{C}, 15 \mathrm{~h}$.

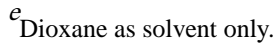

$f_{50}{ }^{\circ} \mathrm{C}, 15 \mathrm{~h}$.

$g_{15 \mathrm{~mol} \%} 12$ 